\title{
Spatial Quantum Correlations Induced by Multiple Scattering of Light
}

\author{
Lodahl, Peter; Lagendijk, Ad
}

Published in:

Conference on Lasers and Electro-Optics and 2006 Quantum Electronics and Laser Science Conference. CLEO/QELS 2006.

Publication date:

2006

Document Version

Publisher's PDF, also known as Version of record

Link back to DTU Orbit

Citation $(A P A)$ :

Lodahl, P., \& Lagendijk, A. (2006). Spatial Quantum Correlations Induced by Multiple Scattering of Light. In Conference on Lasers and Electro-Optics and 2006 Quantum Electronics and Laser Science Conference. CLEO/QELS 2006. (pp. QTUF2). IEEE.

\section{General rights}

Copyright and moral rights for the publications made accessible in the public portal are retained by the authors and/or other copyright owners and it is a condition of accessing publications that users recognise and abide by the legal requirements associated with these rights.

- Users may download and print one copy of any publication from the public portal for the purpose of private study or research.

- You may not further distribute the material or use it for any profit-making activity or commercial gain

- You may freely distribute the URL identifying the publication in the public portal 


\title{
Spatial Quantum Correlations Induced by Multiple Scattering of Light
}

\author{
Peter Lodahl ${ }^{1}$ and Ad Lagendijk ${ }^{2}$ \\ ${ }^{1}$ COM DTU Department of Communications, Optics, and Materials \\ Nano $\bullet D T U$, Technical University of Denmark \\ DTU - Building 345V, DK-2800 Kgs. Lyngby, Denmark \\ Phone: +45 4525 3807, FAX: +4545936581 \\ E-mail:pel@com.dtu.dk \\ ${ }^{2}$ FOM Institute for Atomic and Molecular Physics (AMOLF) \\ Kruislaan 407 Amsterdam, The Netherlands
}

\begin{abstract}
It is experimentally demonstrated how quantum noise of light is transported through a multiple scattering random medium. We predict the existence of novel spatial quantum correlations that are induced by multiple scattering.
\end{abstract}

(C2006 Optical Society of America

OCIS codes: (290.4210), (270.2500), (270.5290)

Coherent transport of light in a disordered random medium has attracted enormous attention both from a fundamental and application point of view. Coherent wave scattering has the potential of enhancing communication capacities, is ubiquitous in acoustical and biomedical imaging, and is the basis for fundamental findings such as intensity correlations, enhanced backscattering, and Anderson localization of light. Recently, theoretical work has considered quantum optics in multiple scattering media and novel fundamental phenomena have been predicted when examining quantum fluctuations instead of merely the intensity of the light [1].

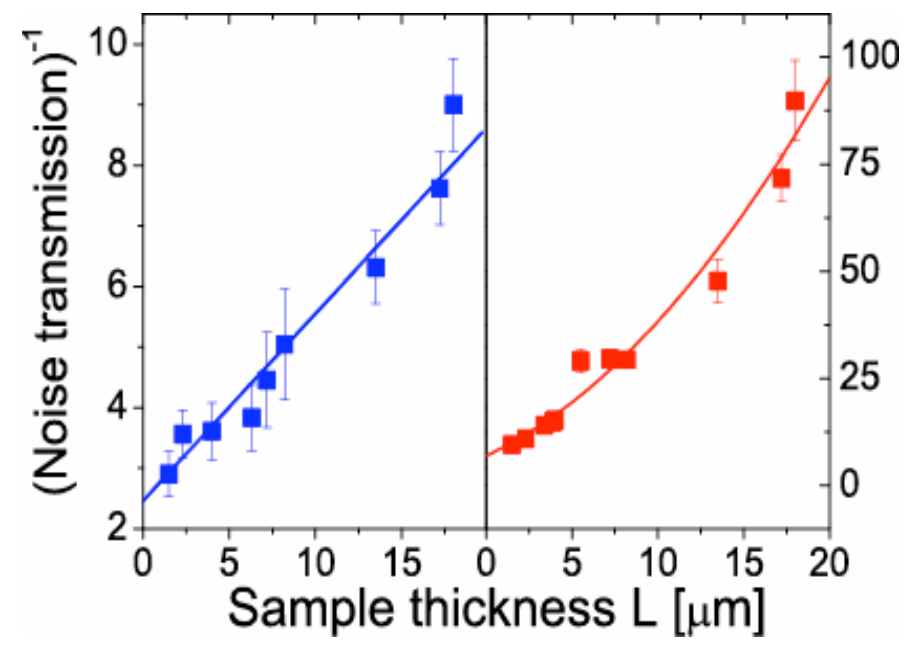

Figure 1. Inverse total transmission of shot noise (left) and technical noise (right) as a function of the thickness of the random medium. The experimental data are well explained by theory (curves).

Here we present the first experimental study of the propagation of quantum noise through a multiple scattering medium composed of elastic scatterers[2]. Two different types of quantum noise measurements have been carried out: total trans- 
mission and short-range frequency correlations. When comparing shot noise (quantum) to technical noise (classical) we observed markedly different behavior, c.f. Fig. 1. The experimental results are found to be in excellent agreement with a quantum model for multiple scattering of light, which allows extracting both static (transport mean free path) and dynamic (diffusion constant) scattering properties of the medium.

When the multiple scattering sample is excited with different quantum states of light (Fock state or thermal state), we predict that novel spatial quantum correlations can be induced by multiple scattering [3,4]. These photon number correlations show that the quantum character of light can be preserved even after many scattering events and after ensemble averaging over different configurations of disorder. The spatial quantum correlation manifests itself in the quantum fluctuations of the total reflection or total transmission through the sample, cf. Fig. 2.

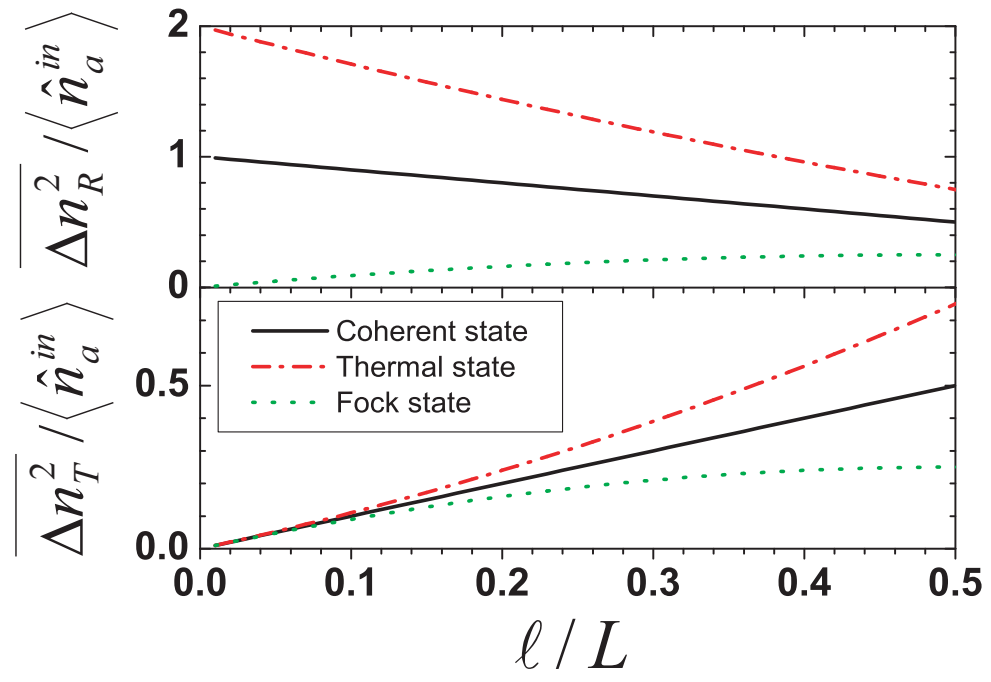

Figure 2. Variance of the quantum fluctuations of the total reflection (upper plot) and total transmission (lower plot) for a coherent state, thermal state and Fock state as a function of the mean free path relative to the sample thickness. Deviations from the linear behavior, which is found for a coherent state, are due to induced spatial quantum correlations.

[1] J. Tworzydlo and C.W.J. Beenakker, “Quantum optical communication rates through an amplifying random medium”Phys. Rev. Lett. 89, 043902, 1-4 (2002).

[2] P. Lodahl and A. Lagendijk, "Transport of quantum noise through random media”, Phys. Rev. Lett. 94, 153905, 1-4 (2005).

[3] P. Lodahl, A.P. Mosk, and A. Lagendijk, "Spatial quantum correlations in multiple scattered light” Phys. Rev. Lett. 95, 173901, 1-4 (2005).

[4] P. Lodahl, "Quantum noise frequency correlations of multiple scattered light", to appear in Opt. Lett. (2006). 